\title{
Lojas on-line de materiais esportivos: fatores influentes na decisão de compra
}

\section{Online sports supplies store: factors that influence purchasing decisions}

\author{
Gustavo Fraile Sordi Mestrando em Administração Pública. Universidade Estadual do Mato Grosso do Sul (UEMS) - \\ Brasil.gustavosordi@uems.br \\ Eduardo Luis Casarotto Doutorando em Administração. Universidade Federal da Grande Dourados (UFGD) - Brasil. \\ eduardocasarotto@ufgd.edu.br \\ Victor Fraile Sordi Doutor em Engenharia e Gestão do Conhecimento. Universidade Federal do Mato Grosso do Sul \\ (UFMS) - Brasil. victor.sordi@ufms.br
}

\begin{abstract}
RESUMO
O fenômeno das compras on-line já foi bastante explorado por pesquisadores no Brasil e no mundo. No entanto, as compras on-line de material esportivo ainda se apresentam como um segmento pouco explorado na literatura. Este artigo teve como objetivo investigar os fatores que influenciam os consumidores a escolher entre as empresas de varejo on-line especializadas em materiais esportivos. Para tanto foram coletados dados a partir de uma survey junto a uma amostra não probabilística, por conveniência, de consumidores universitários. Os resultados indicam que os principais fatores que podem exercer influência na escolha desses consumidores são: a segurança do site, a reputação da marca e/ou loja e o preço. Tais fatores convergem com evidências de outros estudos sobre comportamento de compras on-line, sugerindo que as empresas que atuam neste segmento, ou futuros empreendedores que desejam se inserir no setor, devem investir na construção e fortalecimento da marca e, ou site, praticando preços competitivos, que entreguem valor para o consumidor, proporcionando também uma estrutura de segurança nas plataformas de compras digitais adequada às necessidades dos clientes.
\end{abstract}

Palavras-Chave: Comércio eletrônico. Consumidor on-line. Comportamento do consumidor.

\begin{abstract}
The phenomenon of online shopping has already been widely explored by researchers in Brazil and worldwide. However, the online shopping for sports supplies still presents as a segment little explored in literature. This article aimed to investigate the factors that influence consumers to choose among online retailers specialized in sports goods. For this purpose, data were collected through a survey with a non-probabilistic sample, for convenience, of university consumers. The results indicate that the main factors that may influence the choice of these consumers are: site security, brand and / or store reputation, and price. These factors converge with evidence from other studies on online shopping behavior, suggesting that companies operating in this segment, or entrepreneurs wishing to enter the industry, should invest in the construction and strengthening of the brand and / or the site, have competitive prices, that deliver value to the consumer, while also providing a security structure in the digital purchasing platforms which is adequate to the needs of customers.
\end{abstract}

Keywords: E-commerce. Online consumers. Consumer behavior. 


\section{INTRODUÇÃO}

Nas últimas décadas em paralelo ao crescimento da internet, houve também um crescimento substancial do mercado eletrônico (e-commerce). Segundo EBIT.COM (2018), no principal relatório sobre o comércio eletrônico brasileiro, o Webshopers, o número de consumidores ativos no primeiro semestre de 2018 foi de 27,4 milhões, um crescimento de 7,6\% se comparado ao primeiro semestre de 2017 e um crescimento acumulado de $51,4 \%$ nos últimos cinco anos. Um mercado que faturava aproximadamente $\mathrm{R} \$ 600$ milhões em 2001, chegou à marca de R\$23,6 bilhões somente no primeiro semestre de 2018.

Tais números sugerem que os consumidores brasileiros, assim como no restante do mundo (LIN et al., 2014), estão cada vez mais familiarizados com o comércio eletrônico e que as mudanças não foram somente em relação ao mercado. Os consumidores eletrônicos também apresentam novas características. Para Wilson e Gilligan (2005), esse consumidor é muito mais exigente, menos leal a marcas e empresas e mais disposto a reclamar e buscar seus direitos. Em acréscimo, Dionísio et al. (2009) sugerem que esses consumidores estão cada vez mais bem informados sobre o mercado, uma vez que a informação de que necessita no seu processo de decisão de compra está disponível de forma abundante em múltiplas fontes na internet.

Brandão (2001) ressalta que a internet coloca o poder de controle da informação nas mãos do consumidor, o que dá a ele a possibilidade de decidir em qual momento, onde, no que e qual a quantidade de informação ele irá obter. Essas mudanças no mercado e no perfil dos consumidores demonstram a necessidade de se conhecer melhor esses agentes, no sentido de atender às novas necessidades desses consumidores. O que é uma das funções fundamentais do profissional de marketing (MOWEN; MINOR, 2003).

Uma vez que o propósito do marketing é atender e satisfazer as necessidades e desejos dos consumidores, é necessário conhecer tudo o que envolve o seu comportamento de compra. Entender o que os influencia e o que os leva a escolher uma opção dentre todas as disponíveis para o consumo (KOTLER; KELLER, 2006).

O fenômeno das compras on-line já foi bastante explorado por pesquisadores no Brasil e no mundo (GERALDO; MAINARDES, 2017). No entanto, as compras on-line de material esportivo, mesmo correspondendo a 7,7\% do volume de vendas via e-commerceno país (EBIT.COM, 2018), ainda se apresenta como um segmento pouco explorado na literatura. E a compreensão sobre os fatores que influenciam os consumidores no processo de decisão de compra nesse segmento específico ainda carece de dados empíricos e investigações mais aprofundadas.

Nesse sentido, este estudo foca no comércio eletrônico business-to-consumer(relação entre empresa e consumidor) e tem como objetivo investigar os fatores que influenciam os consumidores a escolher entre as empresas de varejo on-line especializadas em materiais esportivos.

\section{O PROCESSO DE DECISÃO DE COMPRA E O COMÉRCIO ELETRÔNICO}

O comportamento do consumidor representa as ações praticadas pelas pessoas na compra, utilização e descarte dos bens e serviços, levando em conta o que é feito antes, durante e depois da compra (ENGEL; BLACKWELL; MINIARD, 2005). Dessa forma, o consumidor percebe que precisa fazer uma compra para satisfazer uma necessidade ou desejo, dando início assim a um conjunto de etapas que formam o processo de tomada de decisão.

O processo de tomada de decisão de compra é composto por quatro etapas: reconhecimento do problema, busca de informações, avaliação de alternativas e escolha do produto (SOLOMON, 2011). Kotler et al. (2010) acrescenta uma quinta e uma sexta etapa, que seriam o comportamento pós-compra e a fase de descarte.

Além das etapas do processo de tomada de decisão de compra do consumidor, existem os fatores que influenciam esse processo, sendo a análise de tais fatores fundamental para a compreensão deste fenômeno (ENGEL; BLACKWELL; MINIARD, 2005). Viana et al. (2010), com base nos estudos de Kotler e Keller (2006), Churchill e Peter (2003), Mowen e Minor (2003), Solomon (2002) e Engel, Blackwell e Miniard (2005), 
definem como seis os principais fatores que influenciam o processo de tomada de decisão dos consumidores: os fatores culturais, sociais, pessoais, psicológicos, situacionais e fatores de marketing.

Os fatores culturais influenciam o comportamento do consumidor, ao configurar-se como principais determinantes dos desejos e do comportamento de uma pessoa (KOTLER et al., 2010). Na visão de Schiffman e Kanuk (2000, p. 286), pode-se considerar cultura como "a soma total das crenças, valores e costumes aprendidos que servem para direcionar o comportamento de consumo dos membros de determinada sociedade".

Em relação aos fatores sociais, para Kotler et al. (2010), Churchill e Peter (2003) e Kotler e Keller (2006), são constituídos basicamente pelos grupos de referência, família, papéis e status que acabam por influenciar o comportamento do consumidor. Cada papel que uma pessoa desempenha carrega um status, que influencia o comportamento e escolhas do consumidor, assim o indivíduo acaba escolhendo as opções que mais tem relação com o seu papel e status na sociedade.

As necessidades e os desejos das pessoas vão se modificando durante suas vidas (KOTLER et al., 2010). Churchill e Peter (2003) afirmam a existência do ciclo de vida familiar, que é o conjunto de estágios pelos quais as famílias passam, influenciam a identificação de suas necessidades, o modo e a capacidade de satisfazê-las.

A ocupação dos indivíduos também pode influenciar no processo de tomada de decisão de compra. Kotler et al. (2010) afirma que a profissão de cada indivíduo influencia os seus padrões de consumo. 0 consumidor tende a ter um padrão de consumo semelhante aos de pessoas relacionadas pertencentes à mesma ocupação e nível de renda.

Há ao menos quatro fatores psicológicos que influenciam as escolhas dos consumidores: motivação, percepção, aprendizagem e crenças e atitudes (KOTLER; KELLER, 2006). Samara e Morsch (2005) consideram o ambiente físico, o ambiente social, tempo e estados antecedentes como os fatores situacionais que podem influenciar o comportamento do consumidor.

Mowen e Minor (2003) e Churchill e Peter (2003) afirmam que o marketing também pode exercer decisiva influência em qualquer etapa do processo de decisão de consumo. Pode exercer o papel de informar, pode induzir o indivíduo a adquirir algo que talvez nem seja necessário, pode ser decisivo na hora de influenciar o consumidor a escolher o produto ou serviço dentre todas as opções analisadas.

Os fatores supracitados também estão envolvidos no processo de decisão de compra em lojas virtuais, que são o foco desta pesquisa. As transações nessas lojas virtuais constituem o comércio eletrônico. $O$ Comércio Eletrônico (CE) ou e-commerce engloba, segundo Turban e King (2004), todas as atividades de compra ou venda de bens, produtos, serviços ou informações feitas eletronicamente. Em outras palavras, o CE consiste na realização de negócios por meio da Internet (LIMEIRA, 2003).

Em uma definição mais ampla, Cameron (1997) sugere que o CE seria qualquer negócio que seja transacionado eletronicamente por meio de duas entidades de negócio: (1) Business-to-business (entre empresas): tanto os compradores como os vendedores são empresas. Todas as transações eletrônicas efetuadas entre empresas; e (2) Business-to-consumer(entre empresa e consumidor): onde os vendedores são empresas (pessoas jurídicas) e os compradores são indivíduos (pessoas físicas). O`Connel (2002) acrescenta uma terceira forma de CE: (3) Consumer-to-consumer(entre consumidores): que seriam as vendas de produtos ou serviços entre indivíduos (pessoas físicas).

As compras on-line podem trazer benefícios para o consumidor. Segundo Kalakota e Robinson (2002), as principais características e vantagens para os clientes são: a agilidade do atendimento aos clientes; a disponibilidade de poder fazer compras 24 horas por dia e em qualquer localidade que tenha conexão; uma grande variedade de produtos oferecidos pelas empresas em função de não ter a limitação de espaço; e a possibilidade de os consumidores poderem suprir suas necessidades de compra em um único local.

Essas facilidades geradas pelo comércio eletrônico também são citadas por Kotler et al. (2010), que afirma a existência de três principais benefícios para os compradores que utilizam a internet: (1) Conveniência - os clientes podem fazer pedidos de qualquer local que estiverem, a qualquer hora, sem precisar do esforço de se dirigir a uma loja física; (2) Informação - os clientes têm acesso a todo tipo de informação que precisam sobre empresas, produtos e serviços na internet, assim podem fazer comparações para a melhor escolha; e, (3) Comodidade - não é necessário lidar com vendedores e se expor a fatores de persuasão. 
Apesar dos possíveis benefícios que o CE pode trazer, a natureza dessas transações também pode gerar desconfianças por parte do consumidor. Catalani (2006) afirma que um dos maiores medos dos potenciais compradores on-lineé colocar o número do cartão de crédito na internet. Clarke III e Flaherty (2005) apontam outros riscos e desvantagens como a possibilidade de perda de informações pessoais, entrega lenta, potencial de fraude das empresas e a impossibilidade de testar e ver o produto antes da compra.

Para a realização da compra, os consumidores precisam confiar em dois elementos: (1) nas pessoas ou empresas envolvidas e na (2) capacidade da empresa em cumprir sua promessa de venda e de entrega do produto ou serviço desejado (CATALANI, 2006).

As novas tecnologias de segurança e processos têm gerado maior segurança e atratividade para os consumidores. Essa evolução gera maior confiança, o que pode ser observado pelos números crescentes desse mercado (CATALANI, 2006; EBIT.COM, 2018).

Com todos os benefícios e oportunidades de marketing em consequência da popularização da internet, técnicas e práticas estão em constante evolução (KOTLER et al., 2010). As empresas utilizam, por exemplo, os dados de históricos de compras e pesquisas dos consumidores (identificados por tecnologias que identificam os "rastros" deixados na internet) para uma análise e consequente oferta personalizada de um produto (TURBAN; KING, 2004).

Para Limeira (2003), em razão do alcance de informações que o ajudam na escolha do bem ou serviço, o cliente da internet deve ser tratado de forma personalizada. Descobrir como atender e reter estes consumidores é um fator decisivo para as empresas.

Em síntese, as empresas utilizam sites para fortalecer ou para realizar a divulgação em si de seus produtos ou serviços (SPOSITO, 2006). Também os utilizam para a obtenção de uma comunicação mais eficiente e para identificar o comportamento pós-compra dos clientes, suas satisfações e insatisfações, além da possível verificação de tendências para o lançamento de novos produtos.

Dessa maneira, investigar os fatores que influenciam os consumidores a escolher entre as empresas disponíveis no mercado e compreender o processo de decisão de compra na internet, demonstra-se como fundamental nesse contexto de vendas on-line via comércio eletrônico.

\section{PROCEDIMENTOS METODOLÓGICOS}

Para a investigação proposta neste estudo, foi utilizada uma pesquisa survey de caráter quantitativo e fins descritivos (VERGARA, 2010). A pesquisa com fim descritivo é um tipo de pesquisa conclusiva que tem como principal objetivo a descrição de algo (MALHOTRA, 2006), neste caso, a descrição dos principais fatores envolvidos no processo de decisão de compra em lojas virtuais de material esportivo.

Uma pesquisa surveyé uma pesquisa que visa a obtenção de informações e dados sobre ou de um determinado grupo de pessoas, indicado como representante da população-alvo, normalmente por meio de um questionário (PINSONNEAULT; KRAEMER, 1993). O questionário (APÊNDICE A) utilizou-se da escala Likert para medir a percepção dos entrevistados.

A população, público-alvo da pesquisa, foi composta por estudantes universitários. Optou-se por esse recorte, visto que grande parte dos consumidores de material esportivo são graduados ou graduandos, na faixa etária de 20 a 29 anos (EBIT.COM, 2018). O que corresponde com as características do público universitário pesquisado.

A técnica de amostragem, por sua vez, caracterizou-se como não probabilística por conveniência, que consiste numa técnica em que os elementos da amostra são selecionados de acordo com a conveniência. São as pessoas que estão ao alcance dos pesquisadores e dispostos a responder a um questionário (SAMARA; BARROS 2002).

Em virtude da característica da pesquisa, além da praticidade e conforto do respondente, o questionário foi disponibilizado por meio da internet. Para tal, no desenvolvimento e na estruturação do questionário, foi utilizado o software Google Docs. A partir do link gerado pelo software, possibilitou-se a abordagem e disponibilização do questionário diretamente para cada um dos respondentes por meio de $e$ - 
mailou de redes sociais. Os dados foram tabulados, tratados e analisados com auxílio do software Google Docs e do software Excel.

Tendo em vista que a população de universitários, apesar de numerosa, é finita, de acordo com Levine, Bereson e Stephan (2005), para o cálculo de uma amostra de população finita, se utiliza a seguinte fórmula (Figura 1):

Figura 1 - Fórmula para cálculo de amostra com populações finitas.

$$
\mathrm{n}=\frac{\mathrm{Z}^{2} \cdot \hat{\mathrm{p}} \cdot \hat{\mathrm{q}} \cdot \mathrm{N}}{\mathrm{d}^{2}(\mathrm{~N}-1)+\mathrm{Z}^{2} \cdot \hat{\mathrm{p}} \cdot \hat{\mathrm{q}}}
$$

Fonte: Pinsonneault e Kraemer (1993).

Em que " $n$ " é a amostra calculada, "Z" o nível de confiança, "p" a proporção populacional de indivíduos pertencentes à categoria objetivada, " $q$ " a proporção populacional de indivíduos não pertencentes à categoria objetivada, "N" a população e "d" o erro amostral. No cálculo da amostra considerou-se os seguintes valores: o nível de confiança (Z) de 95,5\%; a quantidade de acerto e erro esperados é a padrão, de 50\% cada; e um erro amostral de $10 \%$. Com isso, obteve-se a necessidade de, no mínimo, 100 respondentes. O total de questionários válidos pesquisados foi de 400.

\section{RESULTADOS}

Os resultados estão nas seções seguintes: perfil da amostra, resultados obtidos e marca, reputação, segurança nas compras e preço.

\subsection{Perfil da Amostra}

Conforme disposto na Tabela 1, o perfil dos respondentes foi predominantemente de homens, solteiros, de 20 a 29 anos, com renda mensal até 3 salários mínimos.

Tabela 1 - Perfil da amostra.

\begin{tabular}{|c|c|c|c|c|c|c|c|}
\hline \multirow[t]{2}{*}{ Características } & \multicolumn{7}{|c|}{ Perfil dos respondentes (Percentual) } \\
\hline & \multicolumn{3}{|c|}{ Masculino } & \multicolumn{4}{|c|}{ Feminino } \\
\hline Sexo & & $62 \%$ & & \multicolumn{4}{|c|}{$38 \%$} \\
\hline \multirow{2}{*}{ Faixa Etária } & \multicolumn{3}{|c|}{ De 20 a 29 anos } & \multicolumn{4}{|c|}{ Outros } \\
\hline & & $88 \%$ & & \multicolumn{4}{|c|}{$12 \%$} \\
\hline \multirow{2}{*}{ Estado Civil } & & Solteiros & & \multicolumn{4}{|c|}{ Outros } \\
\hline & & $88 \%$ & & \multicolumn{4}{|c|}{$12 \%$} \\
\hline \multirow[t]{2}{*}{ Renda Mensal } & $\begin{array}{l}\text { De } 1 \text { a } 2 \\
\text { salários } \\
\text { mínimos }\end{array}$ & $\begin{array}{l}\text { De } 2 \text { a } 3 \\
\text { salários } \\
\text { mínimos }\end{array}$ & $\begin{array}{l}\text { Até } 1 \text { salário } \\
\text { mínimo }\end{array}$ & $\begin{array}{l}\text { De } 3 \text { a } 4 \\
\text { salários } \\
\text { mínimos }\end{array}$ & $\begin{array}{l}\text { De } 5 \text { a } 10 \\
\text { salários } \\
\text { mínimos }\end{array}$ & $\begin{array}{l}\text { De } 4 \text { a } 5 \\
\text { salários } \\
\text { mínimos }\end{array}$ & $\begin{array}{l}\text { Acima de } \\
10 \text { salários } \\
\text { mínimos }\end{array}$ \\
\hline & $39,1 \%$ & $21,7 \%$ & $16,3 \%$ & $12 \%$ & $7,6 \%$ & $2,2 \%$ & $1,1 \%$ \\
\hline
\end{tabular}

Fonte: Elaborado pelos autores (2018). 


\subsection{Resultados Obtidos}

Os dados coletados sugerem que os principais fatores considerados pelos consumidores como mais importantes ao escolher entre as lojas virtuais disponíveis são: Segurança, Preço e Reputação da Marca/Site. Conforme disposto na Tabela 2, esses três fatores foram pontuados por mais de $90 \%$ dos respondentes como possuindo um nível de importância maior que 4, sendo 4 considerado como "importante" e 5 como "muito importante" (Escala Likert). A média desses três fatores, conforme destacado na Tabela 2, também foram as maiores com respectivamente: 4,67, 4,56 e 4,62.

Tabela 2 - Fatores influentes na decisão de compra na perspectiva dos respondentes.

\begin{tabular}{lccccccc}
\hline \multirow{2}{*}{ Fatores } & \multicolumn{7}{c}{ Escala de Importância na Percepção dos Consumidores } \\
\cline { 2 - 8 } & $\mathbf{1}$ & $\mathbf{2}$ & $\mathbf{3}$ & $\mathbf{4}$ & $\mathbf{5}$ & $>\mathbf{4}$ & Média \\
\cline { 2 - 8 } & $1,10 \%$ & $0 \%$ & $4,30 \%$ & $18,50 \%$ & $76,10 \%$ & $\mathbf{9 4 , 6 0 \%}$ & $\mathbf{4 , 6 7}$ \\
Segurança do site & $1,10 \%$ & $2,20 \%$ & $4,30 \%$ & $22,80 \%$ & $69,60 \%$ & $\mathbf{9 2 , 4 0 \%}$ & $\mathbf{4 , 5 6}$ \\
Reputação da marca e/ou do site & $0 \%$ & $2,20 \%$ & $6,50 \%$ & $17,40 \%$ & $73,90 \%$ & $\mathbf{9 1 , 3 0 \%}$ & $\mathbf{4 , 6 2}$ \\
Preço & $0 \%$ & $5,40 \%$ & $8,70 \%$ & $26,10 \%$ & $59,80 \%$ & $85,90 \%$ & 4,42 \\
Promoções & $3,30 \%$ & $6,50 \%$ & $14,10 \%$ & $26,10 \%$ & $50,00 \%$ & $76,10 \%$ & 4,15 \\
Valor do frete & $2,20 \%$ & $6,50 \%$ & $21,70 \%$ & $33,70 \%$ & $35,90 \%$ & $69,60 \%$ & 3,90 \\
Opções de produtos & $3,30 \%$ & $6,50 \%$ & $21,70 \%$ & $21,70 \%$ & $46,80 \%$ & $68,50 \%$ & 4,02 \\
Opções de formas de pagamento & $3,30 \%$ & $13 \%$ & $19,50 \%$ & $18,50 \%$ & $45,70 \%$ & $64,20 \%$ & 3,90 \\
Prazo de Entrega & $2,20 \%$ & $5,40 \%$ & $29,30 \%$ & $30,50 \%$ & $32,60 \%$ & $63,10 \%$ & 3,89 \\
Opinião de quem jáy comprou no site & $6,50 \%$ & $9,80 \%$ & $25 \%$ & $26,10 \%$ & $32,60 \%$ & $58,70 \%$ & 3,75 \\
Recomendação de familiares ou amigos & &
\end{tabular}

Fonte: Elaborado pelos autores (2018).

A importância do fator "segurança" encontrada nos dados coletados converge com os resultados da pesquisa apresentada no relatório EBIT.COM (2018), onde a segurança é a principal preocupação apresentada pelos consumidores de lojas virtuais. O fator "preço" nesse mesmo relatório aparece na segunda posição, o que também reforça os resultados encontrados nesta pesquisa. No entanto, esses dados do mercado nacional divergem de dados internacionais como do relatório Retail Consumer (2016) que aponta que a segurança na compra já não é mais uma das principais preocupações nos mercados on-line europeus e americanos.

O preço, a segurança no pagamento e a reputação do site também são destacados no estudo de Botelho et al. (2012) que ao analisar os hábitos de compras dos alunos da graduação de um curso de Administração em uma Universidade do Interior do estado do Paraná, concluem que o preço é o principal item do composto de marketing a influenciar na escolha dos consumidores, seguidos pela segurança no pagamento e a reputação e credibilidade do site.

Os atributos mais bem avaliados no estudo de Santos, Hamza e Nogami (2016), que buscou entender como o consumidor comporta-se perante o consumo de moda na internet e como avalia os atributos da compra, também foram a "Reputação do site" e o "Preço". Deste modo, evidencia-se, com base nos dados coletados, que assim como nos demais segmentos, em lojas on-line de materiais esportivos, os fatores preço, segurança e reputação são essenciais na decisão de compra dos consumidores brasileiros, sobretudo, os consumidores universitários.

\subsection{Marca, Reputação, Segurança nas Compras e Preço}

A marca é definida como um nome, termo, design, símbolo ou qualquer outra característica que identifique o produto e, ou, serviço de um vendedor como distinto daqueles de outros vendedores (CHURCHILL; PETER, 2003). Para Kotler et al. (2010), os benefícios de uma marca forte podem ser de grande valia, já que a marca pode passar a significar algo muito além do que os seus simples produtos. 
Nesse sentido, Nunes e Haigh (2003) destacam que a importância da marca evoluiu em sinergia com o mercado e, ao longo do tempo, passou de apenas um logotipo para diferenciação, até um conjunto de valores tangíveis e intangíveis que diferenciam um produto e, ou, serviço. Esses valores, tanto os tangíveis quanto os intangíveis, podem resultar em uma vantagem competitiva para uma organização (HALL, 1992).

Um desses valores intangíveis de uma marca é a sua reputação. A reputação é um ativo intangível de grande valia para as organizações, um reconhecimento positivamente diferente, é uma grande vantagem competitiva (ROSA, 2006). Ao relacionar o tema reputação com a percepção de segurança, o autor afirma que sem reputação não existe confiança, e sem confiança, não há escolha. Em razão dessa importância, a reputação passou a ser a alma de um negócio, é uma ponte entre a organização e os outros; uma boa reputação é algo muito objetivado (ROSA, 2006).

Ainda sobre marcas e a importância do seu consequente reconhecimento e reputação perante aos consumidores, Keller e Machado (2009) afirmam que na busca pela minimização dos riscos, os consumidores consomem marcas conhecidas, especialmente àquelas que fazem parte de alguma experiência anterior positiva. A marca é um atalho na decisão de compra de um consumidor, pois a marca por si só já informa, não existe necessidade de muitas outras informações prévias e tudo isso minimiza a insegurança do comprador (BAZANINI, 2003). Esse conhecimento e reputação de uma marca, em um mercado globalizado e competitivo, pode ser uma diferenciação determinante para a escolha do comprador (KOTLER et al., 2010).

A importância da reputação também está relacionada com a percepção de segurança que ela pode gerar, esse fator também destacado pelos respondentes é comumente citado em relatórios do setor de $e$ commerce (RETAIL CONSUMER, 2016; EBIT.COM, 2018) e pesquisas acadêmicas (GERALDO; MAINARDES, 2017; QUITÉRIA; MORETTI, 2018).

O medo de colocar o número do cartão de crédito nos sites, a possibilidade de perda de informações pessoais, possibilidades de entregas não realizadas, potencial fraude das empresas, impossibilidade de testar e ver o produto antes da compra, entre outros fatores relacionados a segurança do site/loja são alguns dos aspectos citados na literatura (GERALDO; MAINARDES, 2017; QUITÉRIA; MORETTI, 2018).

Decorrente da constatação da necessidade de percepção de segurança por parte dos consumidores, têm-se trabalhado e evoluído para atender a essa demanda (CATALANI, 2006; CLARKE III; FLAHERTY, 2005). No relatório WebShoppers apurou-se, por meio de interrogações diretas aos compradores de diversas lojas, que o consumidor avalia e dá substancial importância para a gestão de segurança de um site. O relatório aponta que o principal motivo de comprar em uma determinada loja é a confiança na mesma, com 17\% (EBIT.COM, 2018).

A importância da segurança também pode ser observada pela existência de selos de confiabilidade para sites. O supracitado site E-bit, referência em pesquisas e informações sobre o e-commerce nacional, também fornece medalhas de níveis de confiança e satisfação de compra nos sites parceiros (mais de oito mil), quanto melhor o feedback dos compradores, melhor a nota ou nível de confiança da loja.

Outro fator de destaque apontado pelos respondentes foi o preço. Segundo Kotler (2010), o preço funciona como o principal fator determinante na escolha dos compradores. Em conformidade, Rojo (2003) afirma que o preço é o principal atributo considerado para a escolha da loja onde o consumidor fará sua compra. Em complemento Parente (2000), indica que o preço é a variável das ações de marketing que mais rapidamente afeta a competitividade, o volume de vendas, as margens e a lucratividade das empresas.

Os dados apresentados no relatório EBIT.COM (2018) sugerem que o preço, com $16 \%$ das respostas é o segundo fator mais importante na hora de escolher o site para compras. Também é o segundo principal motivo para não se fazer compra em um site, com 14\% das respostas.

Apesar de ser uma variável importante das práticas de marketing, o preço não é um conceito absoluto, nem sempre a percepção de melhor valor para a compra tem as mesmas características e significados. Os consumidores procuram um bom valor no que adquirem, para alguns indivíduos um bom valor significa um preço baixo, mas outros estão dispostos a pagar mais se existir a percepção de obtenção de um valor maior de qualidade (LEVY; WEIZ, 2000). Kotler (2010) também discorre sobre o tema, para ele os clientes dão preferência ao produto, serviço ou marca que proporcionam um maior valor. O valor entregue ao cliente depende do desempenho da oferta em relação a suas expectativas e não necessariamente do preço. 
Entretanto, no relatório Retail Consumer (2016), uma pesquisa com consumidores realizada pelo PwC (2016) contém informações valiosas. Essa pesquisa que contou com mais de 22 mil entrevistados de diversos países, indica que $60 \%$ dos consumidores escolhe o varejista com base no preço. Mais especificamente no contexto do Brasil, o relatório mostrou que $58,7 \%$ dos consumidores brasileiros afirmam que o fator de maior relevância na escolha de lojas varejistas de sua preferência é um bom preço, seguido pela confiança na marca do produto (outro fator de destaque no presente estudo), citada por $44,6 \%$ dos entrevistados.

Preço, Reputação da marca e Segurança do site são três elementos que fazem parte das ações de marketing aos consumidores por parte das empresas, entretanto também se encaixam em outras classificações (KOTLER et al., 2010). Isto é, o preço pode ser classificado como um fator econômico enquanto a reputação e a segurança do site estão, igualmente, relacionadas com fatores sociais, pessoais e psicológicos, já que essas percepções estão relacionadas, por exemplo, com as experiências passadas, as opiniões pessoais ou de grupos e às crenças dos consumidores (KOTLER, 2010).

Em relação às etapas do processo de decisão em que esses fatores podem impactar, o preço já está inserido no contexto da busca por informações, nesta fase o consumidor já pode levar em conta o que é por ele considerado como um bom valor de preço e assim já excluir ou adicionar opções para a sua aquisição (SOLOMON, 2002). O preço também pode ser influente nas etapas de avaliação das alternativas e da decisão de compra, já que no momento de análise, o preço pode exercer uma grande influência na escolha da melhor opção de compra (CHURCHILL; PETER, 2003).

Entretanto, a percepção de reputação ou segurança do site e/ou marca pode fazer com que o consumidor pule a etapa de busca por informações. Como já discutido, uma boa reputação e, ou reconhecimento da marca ou site pode gerar uma confiança e fidelidade que já pode definir a opção de compra (BAZANINI, 2003). Portanto, a reputação e a segurança do site e, ou marca podem ser ainda mais impactantes nas etapas de avaliação das alternativas e da efetivação da decisão de compra.

\section{CONSIDERAÇõES FINAIS}

Frente aos desafios do e-commerce, este trabalho teve como objetivo investigar os fatores que influenciam os consumidores a escolher entre as empresas de varejo on-line especializadas em materiais esportivos. Entre os respondentes, universitários, predominaram os do sexo masculino, com idades que se enquadram na faixa entre 20 e 29 anos, solteiros, e que possuem renda mensal de até 3 salários mínimos.

De forma agregada, os resultados obtidos apontaram que na percepção do consumidor, os três fatores que mais influenciam na decisão de compra, são: (1) a segurança do site; (2) a reputação da marca e, ou site; e (3) o preço.

O preço pode ser classificado como um fator econômico enquanto a reputação e a segurança do site estão, igualmente, relacionadas a fatores sociais, pessoais e psicológicos, já que essas percepções podem estar relacionadas com as experiências passadas, as opiniões pessoais ou de grupos e às crenças dos consumidores.

Dessa forma, os resultados da pesquisa indicam que as empresas que atuam neste segmento, ou futuros empreendedores que desejam se inserir no setor, devem investir na construção e fortalecimento da marca e/ou site, praticando preços competitivos, que entreguem valor para o consumidor, proporcionando também uma estrutura de segurança nas plataformas de compras digitais adequada às necessidades dos clientes.

Como a pesquisa direcionou-se apenas a consumidores universitários, sugere-se novas pesquisas de natureza qualitativa que busquem se aprofundar no entendimento do comportamento de compra dos consumidores neste segmento específico, assim como pesquisas quantitativas com amostras mais abrangentes, visando contribuir com mais evidências sobre o fenômeno estudado.

\section{REFERÊNCIAS}

BAZANINI, R. Marketing e imagem corporativa na perspectiva da rhetorical criticism. São Paulo: Plêiade, 2003. 
BOTELLHO, Grace Kelly Novais; OLIVEIRA, GOMES de, Eveline; SILVA, Hermes Moretti Ribeiro da. E-commerce: análise do hábito de compra de jovens universitários em lojas virtuais em relação ao composto de marketing. In: ENCONTRO CIENTÍFICO DE ADMINISTRAÇÃO, ECONOMIA E CONTABILIDADE (ECAECO), 2., 2012, Ponta Porã, Mato Grosso do Sul. Anais [...]. Ponta Porã: UEMS, 2012.

BRANDÃO, V.C. Comunicação e marketing na era digital: a internet como mídia e canal de vendas. In: CONGRESSO BRASILEIRO DE CIÊNCIAS DA COMUNICAÇÃO (INTERCOM), 14., 2001, Campo Grande, Mato Grosso do Sul. Anais do XXIV INTERCOM, v. 1, n. 1, Campo Grande: Uniderp, 2001.

CAMERON, D. Electronic commerce: the new business platform of the Internet. Charleston: Computer Technology Research, 1997.

CATALANI, L. et al. E-commerce. 2. ed. Rio de Janeiro: FGV, 2006.

CHURCHILL JR, G. A.; PETER, J. P. Marketing: criando valor para os clientes. 2. ed. São Paulo: Saraiva, 2003.

CLARKE III, I.; FLAHERTY, T. B. Advances in eletronic marketing. Hershey: IGI Publishing. 2005.

DIONÍSIO, P. et al. B-Mercator: blended marketing. Lisboa: Publicações Dom Quixote, 2009.

EBIT.COM. Relatório webshoppers 2018. Disponível em: http://www.ebit.com.br/webshoppers. Acesso em 2 out. 2018.

ENGEL, J. F.; BLACKWELL, R. D.; MINIARD, P. W. Comportamento do consumidor. Rio de Janeiro: LTC, 2005.

GERALDO, G. C.; MAINARDES, E. W. Estudo sobre os fatores que afetam a intenção de compras online. REGERevista de Gestão, São Paulo, v. 24, n. 2, p. 181-194, 2017.

HALL, R. The strategic analysis of intangible resources. Strategic Management Journal, v. 13, n. 2, p. 135$144,1992$.

KALAKOTA, R.; ROBINSON, M. M-business tecnologia móvel e estratégia. Porto Alegre: Bookman, 2002.

KOTLER, Philip; KELLER, Kevin Lane. Administração de marketing: a bíblia do marketing. 12. ed. São Paulo: Saraiva, 2006.

KELLER, K. L.; MACHADO, M. Gestão estratégica de marcas. São Paulo: Pearson Prentice Hall, 2009.

KOTLER, P. et al. Marketing 3.0: as forças que estão definindo o novo marketing centrado no ser humano. Rio de Janeiro: Elsevier, 2010.

LEVINE, D. M.; BERENSON, M. L.; STEPHAN, D. Estatística: teoria e aplicações-usando Microsoft Excel português. Rio de Janeiro: LTC, 2005.

LEVY, M.; WEIZ, B. A. Administração de varejo. São Paulo: Atlas, 2000

LIMEIRA, T. M. V. E-marketing: o marketing na internet com casos brasileiros. São Paulo: Saraiva, 2003.

LIN, Jiabao et al. Understanding the evolution of consumer trust in mobile commerce: a longitudinal study. Information Technology and Management, v. 15, n. 1, p. 37-49, 2014.

MALHOTRA, N. Pesquisa de marketing: uma orientação aplicada. 4. ed. Porto Alegre: Bookman, 2006.

MOWEN, J. C.; MINOR, M. S. Comportamento do consumidor. São Paulo: Prentice Hall, 2003.

NUNES, G.; HAIGH, D. Marca: valor do intangível medindo e gerenciando seu valor econômico. São Paulo: Atlas, 2003. 
O'CONNEL, Brian. B2B. Com: ganhando Dinheiro no E-Commerce Business-to-Business. São Paulo: Makron Books, 2002.

PARENTE, J. Varejo no Brasil: gestão e estratégia. São Paulo: Atlas, 2000.

PINSONNEAULT, A.; KRAEMER, K. L. Survey research methodology in management information system: an assessment. Journal of Management Information Systems, v. 10, n. 2, p. 75-105, 1993.

PWC. Relatório total retail consumer 2016. Disponível em: https://www.pwc.ru/en/publications/totalretail2016.html. Acesso em: 10 jun. 2019.

QUITÉRIA, Cristina; MORETTI, Sergio Luiz do Amaral. Relacionamento, facilidade de uso percebida, comunicação boca a boca e hospitalidade virtual em compras online: validação de um modelo de pesquisa. Revista Brasileira de Marketing, São Paulo, v. 17, n. 4, p. 620-636, 2018.

ROJO, F. J. G. Varejo. In: DIAS, S. R. (org.). Gestão de marketing. São Paulo: Saraiva, 2003. p. 361-394.

ROSA, M. A reputação na velocidade do pensamento: imagem e ética na era digital. São Paulo: Geração Editorial, 2006.

SAMARA, B. S.; BARROS, J. C. Pesquisa de marketing: conceitos e metodologias. São Paulo: Prentice Hall, 2002.

SAMARA, B. S.; MORSCH, M. A. Comportamento do consumidor: conceitos e casos. São Paulo: Prentice Hall, 2005.

SANTOS, Renata Carneiro; HAMZA, Kavita Miadaira; NOGAMI, Vitor Koki da Costa. E-commerce de Artigos de Moda: Análise da Influência dos Atributos da Compra Online. Revista Interdisciplinar de Marketing, v. 5, n. 1, p. 64-80, 2016.

SCHIFFMAN, L. G.; KANUK, L. L. Comportamento do consumidor. 6. ed. Rio de Janeiro: LTC, 2000.

SOLOMON, M. R. O comportamento do consumidor: comprando, possuindo e sendo. 5. ed. Porto Alegre: Bookman, 2002. 2011.

O comportamento do consumidor: comprando, possuindo e sendo. 9. ed. Porto Alegre: Bookman,

SPOSITO, E. S. Redes e cidades. São Paulo: Ed. UNESP, 2006.

TAVARES, M. C. A força da marca: como construir e manter marcas fortes. São Paulo: Harbra, 1998.

TURBAN, E.; KING, D. Comércio eletrônico: estratégia e gestão. São Paulo: Pearson Prentice Hall, 2004.

VERGARA, S. C. Projetos e relatórios de pesquisa em administração. 12. ed. São Paulo: Atlas, 2010.

VIANA, J. J. S. et al. A importância do comportamento de compra do consumidor para o merchandising: uma reflexão teórica. In: CONGRESSO INTERNACIONAL DE ADMINISTRAÇÃO (ADMPG), 7., 2010, Ponta Grossa, Paraná. Anais do VII ADMPG. Ponta Grossa: Cine Teatro Opera, 2010.

WILSON, R.; GILLIGAN, C. Strategic marketing management: planning, implementation and control. 3. ed. Rio de Janeiro: Elsevier, 2005. 


\section{APÊNDICE A - QUESTIONÁRIO}

*Obrigatório

1. Você está matriculado em algum curso de graduação ou pós-graduação? *

$\begin{array}{lll}0 & \text { Sim } \\ 0 & t^{+-} \quad \text { Não }\end{array}$

2. Faz ou já fez compras pela internet? *

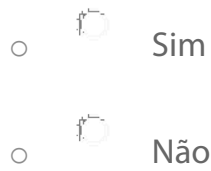

3. Costuma comprar ou já realizou compras em lojas de materiais esportivos na internet? * Exemplo: Netshoes, Centauro, Adidas, Nike, entre outros.

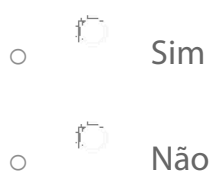

4. Sexo:
- $t^{+-}$Masculino
$0 \quad$ Feminino

5. Sua faixa etária:
- Até 19 anos
De 20 a 29 anos
- De 30 a 39 anos
$+\quad$ De 40 a 49 anos
$0 \quad$ De 50 a 59 anos
- $\quad$ Acima de 60

6. Estado civil:
$\circ \quad$ Solteiro(a)
- Casado(a) 


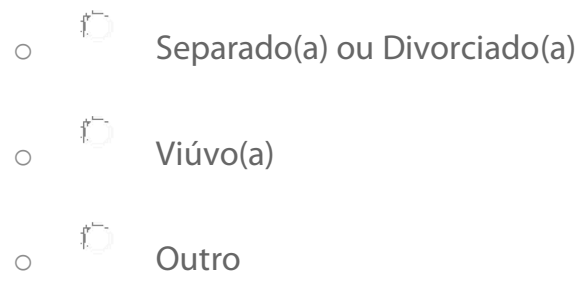

\section{Sua renda mensal:}

Salário mínimo em 01/01/2015 = R\$788,00

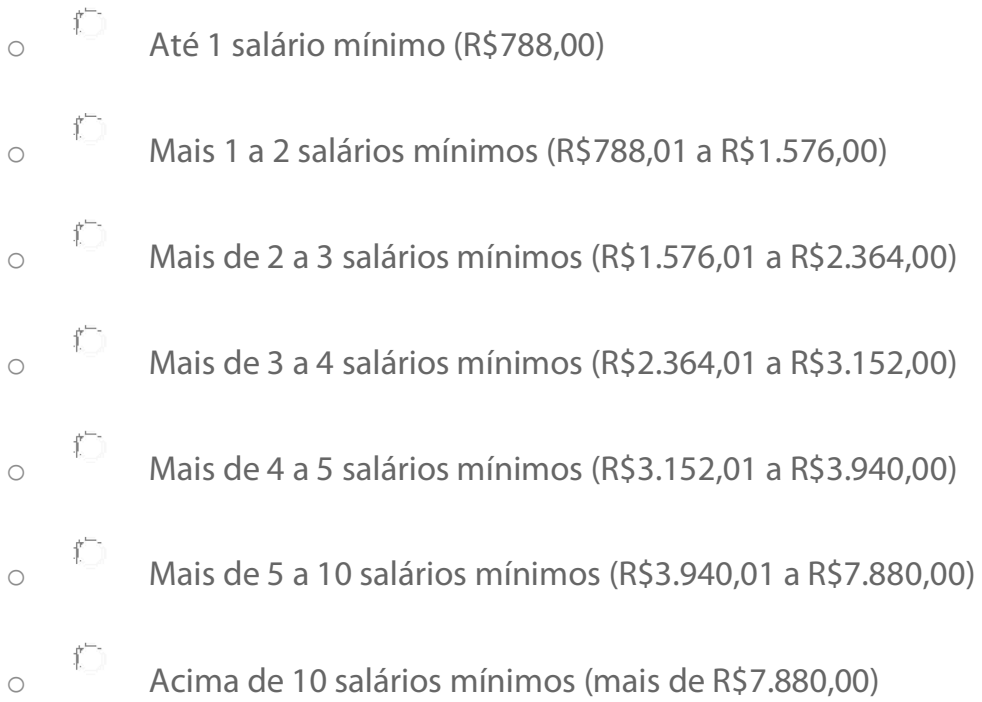

Existem vários sites nos quais você pode realizar essa compra, o que te faz escolher um deles? Serão citados alguns fatores que podem influenciá-lo na hora da compra. Responda as questões a seguir classificando a importância desses fatores na sua decisão. A pontuação varia de 1 a 5, sendo que quanto mais próximo de 1, menor a importância, e quanto mais próximo de 5, maior a importância.

\section{Reputação da Marca e/ou do site.}

$\begin{array}{lllll}1 & 2 & 3 & 4 & 5 \\ t^{2} & t & t & t & t\end{array}$

\section{Segurança do site.}

$\begin{array}{lllll}1 & 2 & 3 & 4 & 5 \\ t & t & t & t\end{array}$

\section{Opinião de quem já comprou no site.}

Exemplo: Conversas ou nos comentários pós-compra. 


$\begin{array}{lllll}1 & 2 & 3 & 4 & 5 \\ t^{2} & t & t & t & t\end{array}$

11. Recomendação de familiares ou amigos.

$\begin{array}{lllll}1 & 2 & 3 & 4 & 5 \\ t^{*} & t & t & t & t\end{array}$

12. Opções de produtos.

Quantidade de opções de produtos que o site oferece.

$\begin{array}{lllll}1 & 2 & 3 & 4 & 5 \\ t^{*} & t & t & t & t\end{array}$

\section{Promoções.}

$\begin{array}{lllll}1 & 2 & 3 & 4 & 5 \\ t & t & t\end{array}$

14. Preço.

$\begin{array}{lllll}1 & 2 & 3 & 4 & 5\end{array}$

15. Opções de formas de pagamento.

$\begin{array}{lllll}1 & 2 & 3 & 4 & 5 \\ t & t & t & t\end{array}$

16. Valor do frete.

$\begin{array}{lllll}1 & 2 & 3 & 4 & 5\end{array}$

it $\quad t 5$ to


17. Prazo de entrega.

$\begin{array}{lllll}1 & 2 & 3 & 4 & 5\end{array}$

$t^{2} \quad t \quad t$

Enviar 